\title{
DEFORMATION AT THE LIMIT OF ELASTIC STABILITY
}

\author{
J.W. Morris, Jr. ${ }^{1}$, C.R. Krenn ${ }^{1}$, D. Roundy ${ }^{2}$ and Marvin L. Cohen ${ }^{2}$ \\ ${ }^{1}$ Department of Materials Science and ${ }^{2}$ Department of Physics, \\ University of California, and \\ Materials Sciences Division, Lawrence Berkeley National Laboratory \\ Berkeley, California 94720 \\ phone: 510486 6482; fax: 510486 4023; email: jwmorris@uclink4.berkeley.edu
}

\begin{abstract}
We describe mechanical behavior in a defect-free world and find many familiar features. Among them: (1) In a world governed by elastic stability, the common bcc metals would exhibit "pencil glide" in $\langle 111>$ and would cleave on $\{100\}$. They would be "conditionally brittle" at low temperature. (2) The common fec metals would glide on $\{111\}$ and would not cleave under simple tensile loads. They would be ductile at low temperature. (3) The maximum values of the nanohardness of simple metals would be very nearly what they are.
\end{abstract}

\section{INTRODUCTION}

We think it appropriate that a conference devoted to the status of the dislocation theory contains at least one paper that ruminates on what the world would be like if there were no such thing: if the world were a theorist's dream (though a metallurgist's nightmare) in which all solids were perfect crystals undisturbed by defects of any kind. In such a world solids would shear, and they would break in tension, but their strengths would be governed by the elastic stability of the lattice rather than the critical resolved shear stress.

There are, in fact, several practical reasons to be interested in behavior at the limit of elastic stability [1]. First, elastic instability defines the ideal limit of strength [2-4], and it is sometimes useful to know the highest strength a particular material can possibly have. Second, the elastic limit is actually reached, or, at least, closely approached in a number of experimental situations. The most common case is deformation via stressinduced phase transformations, as in certain austenitic steels and in Si in indentation. However, even normal, ductile metals seem to approach the limit of strength in nanoindentation experiments, and stronger alloys may also do so in the region of stress concentration ahead of a crack. Finally, elastic instability is one of the few problems in solid mechanics that can actually be solved ab initio. Existing pseudopotential codes are capable of following elastic deformation to the point of instability with reasonable accuracy, and a number of calculations have been done [1,5-8].

The authors have been studying the limits of strength for some years now. In the course of that investigation we have often been surprised both by the simplicity and the 
familiarity of behavior at the limit of strength. A surprising variety of mechanical behaviors that are ordinarily attributed to the peculiarities of dislocations would also be found in a defect-free world at low temperatures at which thermal activation is unimportant. In the limited space available here we discuss three examples. (1) In a defect-free world, the common bcc metals would exhibit "pencil glide" in $<111>$ and would cleave on $\{100\}$. They would be "conditionally brittle". (2) The common fcc metals would glide on $\{111\}$ and would not cleave under simple tensile loads. They would be ductile. (3) The maximum values of the nanohardness of simple metals would be very nearly what they are.

\section{BCC METALS}

Computations of the ideal tensile strengths of unconstrained bcc metals show that they are weakest when pulled in a $<100>$ direction, and would, therefore, be expected to cleave on $\{100\}$ planes. Ab initio calculations $[1,9,10]$ give an ideal tensile strength of about $30 \mathrm{GPa}$ for $\mathrm{W}\left(0.07 \mathrm{E}_{100}\right)$, and an approximate value around $10.5 \mathrm{GPa}$ for $\mathrm{Fe}$ $\left(0.08 \mathrm{E}_{100}\right)$ where $\mathrm{E}_{100}$ is the tensile modulus in the $<100>$ direction.

There is a simple crystallographic argument that explains both the cleavage plane and the ideal tensile strength (Fig. 1). Applying a uniaxial tensile stress along $<100>$ carries the bcc structure into an fcc structure with the same volume at a tensile strain of 0.26 (the "Bain strain"). By symmetry, both structures are unstressed, so the tensile stress must pass through at least one maximum along the transformation path, at a critical strain much less than 0.26 . If we assume a single maximum (that is, if fcc symmetry produces an energy maximum along the strain path) and fit the stress-strain curve with a sinusoid that has the correct modulus at low strain, the tensile strength in $\langle 100>$ is given by

$$
\sigma_{\mathrm{m}} \approx 0.08 \mathrm{E}_{100}
$$

in agreement with the ab initio calculations.

The ideal shear strength of a bcc metal also reflects its symmetry. In our study of tungsten [9], we calculated the ideal strengths of all of the experimentally observed slip systems in bcc metals. The strength for slip in a $<110>$ direction on a $\{110\}$ plane was 19.3 GPa. The strengths for relaxed shear (uniaxial stress) in the $<111>$ direction on the
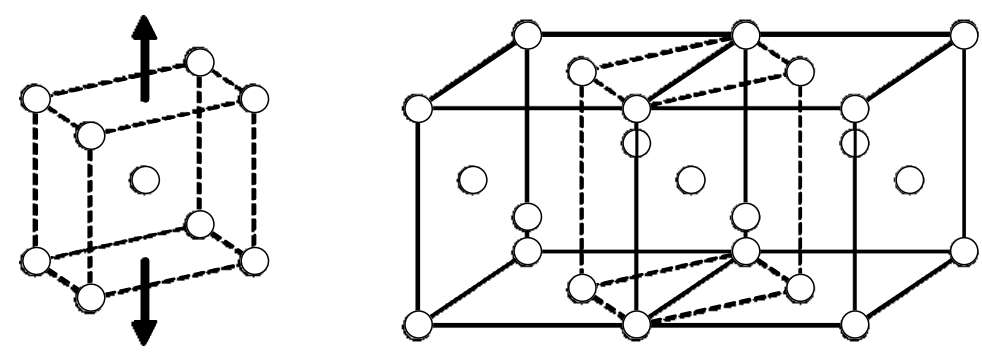

Fig. 1: The Bain strain connecting the bcc and fcc structures. If bcc is pulled in tension on [001] while contracting along [100] and $[010]$ it generates an fcc crystal as shown. 
$\{110\},\{112\}$ and $\{123\}$ planes were almost identical: $\tau_{\mathrm{m}} \approx 17.7 \mathrm{GPa} \approx 0.11 \mathrm{G}_{111}$, where $\mathrm{G}_{111}$ is the shear modulus for shear in the $<111>$ direction. While we did not check all possible slip systems, it seems very probable that the minimum should involve shear in a close packed direction, and it is likely that the minimum strength for any slip system in bcc will be $\approx 0.11 \mathrm{G}_{111}$. In all three cases the shear strain at instability is about 0.17 . A calculation for Mo gives similar results, with $\tau_{\mathrm{m}} \approx 15.6 \mathrm{GPa} \approx 0.12 \mathrm{G}_{111}$. The symmetry rule that governs this case is illustrated in Fig. 2. Essentially, a shear in the $<111>$ direction tilts planes that are perpendicular to the $<111>$ axis. If we allow relaxation of the atom positions in these planes, they come into an atomic registry that changes the crystal symmetry at a shear strain of $\approx 0.34$, irrespective of the plane of tilt (the shear plane). There is, hence, a maximum in the shear strength at about half this shear, 0.17. If we fit the stress-strain relation with a sinusoid that gives the correct modulus in the elastic limit, we obtain

$$
\tau_{\mathrm{m}} \approx 0.11 \mathrm{G}
$$

in good agreement with the ab initio calculations.

A number of bcc metals have similar strengths for slip in the $<111>$ direction on various planes, a phenomenon that is known as "pencil glide" and is attributed to the peculiarities of dislocation glide in bcc. These calculations show that defect-free crystals would behave in a very similar way.

The balance between the shear and tensile strengths suggested by eqs. (1) and (2) is such that, in a defect-free world, the common bcc metals would cleave if loaded along $<100>$, but not if loaded in other directions. Taking $\mathrm{W}$ as an example, a uniaxial load along $<100>$ would reach the ideal cleavage strength, $\approx 30 \mathrm{GPa}$, when the shear stress in the most favorable slip system was only around $14 \mathrm{GPa}$, below the ideal shear strength. However, a uniaxial load along $<111>$ or $<110>$ would cause the shear strength to be exceeded before the tensile stress in $\langle 100>$ reached the ideal value. A single crystal would be ductile or brittle, depending on the direction of the load.
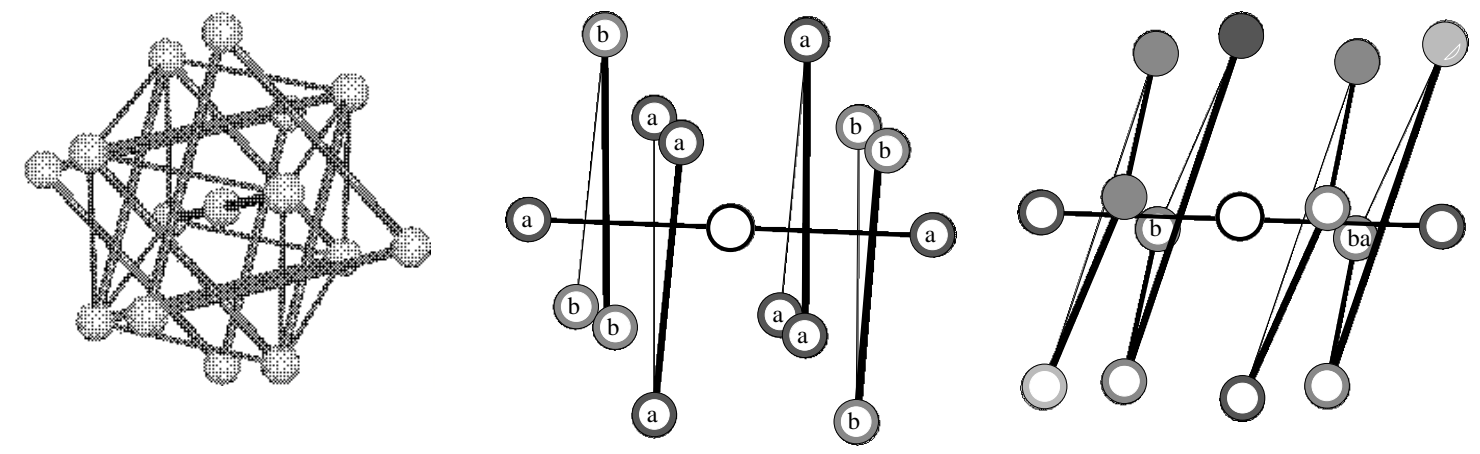


\section{FCC METALS}

The tensile strength of defect-free fcc crystals could, in theory, also be governed by the Bain strain. As illustrated in Fig. 3, an fcc crystal can be converted into bec by straining in tension in the [110] direction. The tensile strain required to reach bcc is, in fact, relatively small, so the estimated strength would be small as well. However, reaching bcc from fcc with a [110] pull requires very substantial relaxations in the perpendicular directions. The crystal must expand equally along [1-10], and contract to an even greater degree along [001]. These large relaxations are inconsistent with the Poisson contractions of typical fcc metals in the linear elastic limit. Fcc metals do not start out along this deformation path when pulled along [110] and, apparently, never find it.

Nonetheless, the [110] direction does seem to be the weak direction for tension in fcc. Defect-free fcc metals have very high tensile strengths when pulled along [100] or [111], but are relatively weak when pulled along [110]. Our own recent (unpublished) $a b$ initio calculations of $\mathrm{Al}$ and $\mathrm{Cu}$ give [110] tensile strengths of $5.2 \mathrm{GPa}\left(0.07 \mathrm{E}_{110}\right)$ and 6.5 $\mathrm{GPa}\left(0.05 \mathrm{E}_{110}\right)$ respectively. (These numbers are reasonably close to those Milstein and Chantasiriwan [11] found from an "embedded atom" model, but the $\mathrm{Cu}$ value is very different from that published by Sob, et al. [10]. The latter authors apparently did not allow asymmetric relaxation in the plane perpendicular to [110].) The common weakness in the $<110>$ directions suggests an extremum dictated by symmetry, but we have not yet identified it.

By contrast, the shear behavior of defect-free fcc crystals is conventional. The weak directions in shear are $<112>$ directions in $\{111\}$ planes, as one would expect from a rigid-ball model of the close-packed fcc structure. There is significant relaxation in the plane as the shear approaches instability, which is significantly different in character for $\mathrm{Al}$ and $\mathrm{Cu}$ [12]. However, in both cases [8] the ideal shear strength is about $0.08 \mathrm{G}_{111}$ : $1.85 \mathrm{GPa}\left(0.084 \mathrm{G}_{111}\right)$ for $\mathrm{Al}, 2.65 \mathrm{GPa}\left(0.088 \mathrm{G}_{111}\right)$ for $\mathrm{Cu}$.

The shear strengths of $\mathrm{Al}$ and $\mathrm{Cu}$ are low enough to insure that defect-free material will be ductile under normal conditions. Even though the ideal tensile strengths of these crystals are not very high when they are pulled along $<110>$, the shear stresses on

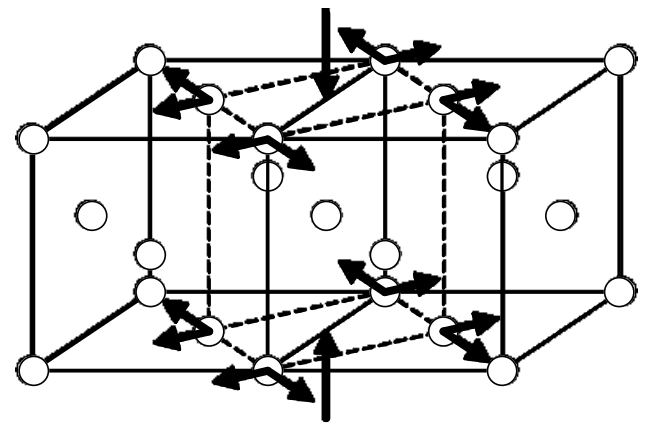

Fig. 3: Bain strain of an fcc lattice through tension along [110]. The crystal must expand equally along [1-10], and contract dramatically along [001]. 
the most favorably oriented slip systems are exceeded well before the ideal tensile strength is reached.

\section{NANOINDENTATION}

A nanoindentation test is, essentially, a microhardness test done with a nanotipped indenter. Until the substrate yields, the deformation field of the indenter should be approximately Hertzian, which makes it possible to use the data to infer the stresses and strains at which yielding occurred. Moreover, since the maximum shear in a Hertzian strain field is well beneath the surface, nanoindentation tests can sample defect-free volumes, and may, therefore, test the ideal strength.

Surprisingly, the shear strengths inferred from recent nanoindentation tests substantially exceed the computed ideal strengths. Thus, Bahr, et al. [13] report data showing shear stresses as high as $28 \mathrm{GPa}$ in W prior to yielding, well beyond the value $(18 \mathrm{GPa})$ that corresponds to the ideal strength on any of the common slip planes. Nix [14] reported preliminary Mo data giving a maximum strength of $23 \mathrm{GPa}$, compared to the theoretical shear strength of $15.6 \mathrm{GPa}$.

The discrepancy between these values is largely removed if one recognizes that the Hertzian stress field is modified by non-linearity as the ideal strength is approached. If we assume (on geometric grounds) that the Hertzian solution gives a reasonable approximation to the strain field, but, because of the softened moduli, mis-estimates the stress at the instability, and use sinusoidal stress-strain dependence, we obtain the maximum shear stress

$$
\tau_{\mathrm{m}}=\left[\frac{2}{\pi}\right] \tau_{\mathrm{H}}=0.64 \tau_{\mathrm{H}}
$$

where $\tau_{\mathrm{m}}$ is the ideal shear strength and $\tau_{\mathrm{H}}$ is the instability stress inferred from the linear elastic Hertzian stress field. A preliminary finite-element calculation using a sinusoidal stress-strain relation confirms the small perturbation of the Hertzian strain field, but

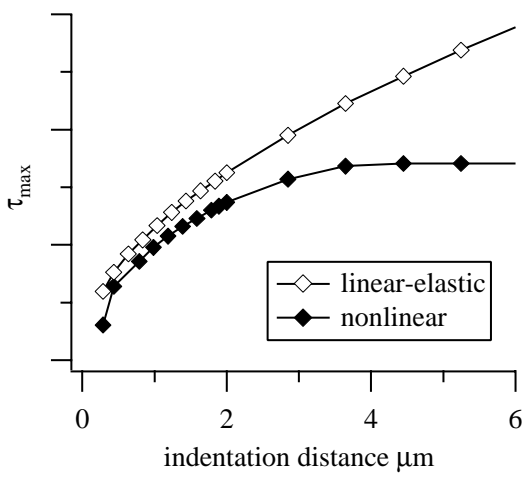

Fig. 4: $\quad$ Maximum $\tau$ under a rigid indenter, with linear and sinusoidal stress-strain relations. When the shear stress of the sinusoidal material hits maximum, it is about $0.69 \tau_{\text {linear. }}$ 
shows the large effect on the stress field (Fig. 4). In this case, $\tau_{\mathrm{m}} \approx 0.69 \tau_{\mathrm{H}}$, in reasonable agreement with eq. 3. Using eq. (3), the measured shear strength of $\mathrm{W}$ drops to $17.8 \mathrm{GPa}$ while that of Mo drops to $14.6 \mathrm{GPa}$, in good agreement with the theoretical estimates.

\section{ACKNOWLEDGEMENTS}

The authors appreciate helpful discussions with Daryl Chrzan, Dept. of Materials Science, and with Steven Louie and Seung-Hoon Jhi, Dept. of Physics, Univ. of California, Berkeley. This work was supported by the Director, Office of Energy Research, Office of Basic Energy Sciences, U.S. Department of Energy, under Contract No. DE-AC03-76SF00098, and by the National Science Foundation Grant No. DMR9520554.

\section{REFERENCES}

1. J.W. Morris, Jr, C.R. Krenn, D. Roundy and M. L. Cohen), in Phase Transformations and Evolution in Materials, P.E. Turchi and A. Gonis, eds., TMS, Warrendale, Pa, 2000, pp. 187-208

2. R. Hill and F. Milstein, Phys. Rev. B, 15, 3087-97 (1977)

3. J. Wang, J. Li, S. Yip, S. Phillpot and D. Wolf, Phys. Rev. B, 52, 12,627-35 (1995)

4. J.W. Morris, Jr. and C.R. Krenn, Phil. Mag. A (in press)

5. A.T. Paxton, P. Gumbsch and M. Methfessel, Phil. Mag. Lett., 63, 267-274 (1991)

6. W. Xu and J. A. Moriarty, Phys. Rev. B., 54, 6941-51 (1996)

7. M. Sob, L.G. Wang and V. Vitek, Mat. Sci. Eng., A234-236, 1075-78 (1997)

8. D. Roundy, C.R. Krenn, M.L. Cohen and J.W. Morris, Jr., Phys. Rev. Lett., $\underline{82}$, 2713-16 (1999)

9. D. Roundy, C.R. Krenn, M.L. Cohen and J.W. Morris, Jr., LBNL Rept. \#44373, (1999) (in review)

10. M. Sob, L.G. Wang and V. Vitek, Kovove Materialy, 36, 145-52 (1998)

11. F. Milstein and S. Chantasiriwan, Phys. Rev. B, 58, 6006-18 (1998)

12. C.R. Krenn, D. Roundy, J.W. Morris, Jr. and M.L. Cohen, Mat. Sci. Eng. A (in press))

13. D.F. Bahr, D.E. Kramer and W.W. Gerberich, Acta Mater., 46, 3605-17 (1998)

14. W.D. Nix, Dept. Materials Science, Stanford Univ., Private Communication, 1999 